2011-06

Learning associations between action and perception: Effects of incompatible training on body part and spatial priming

\title{
Wiggett, AJ
}

http://hdl.handle.net/10026.1/17637

10.1016/j.bandc.2011.02.014

Brain and Cognition

Elsevier BV

All content in PEARL is protected by copyright law. Author manuscripts are made available in accordance with publisher policies. Please cite only the published version using the details provided on the item record or document. In the absence of an open licence (e.g. Creative Commons), permissions for further reuse of content should be sought from the publisher or author. 


\title{
Learning associations between action and perception: Effects of incompatible training on body part and spatial priming
}

\author{
Alison J. Wiggett ${ }^{\mathrm{a}, *}$, Matt Hudson ${ }^{\mathrm{b}}$, Steve P. Tipper ${ }^{\mathrm{a}}$, Paul E. Downing ${ }^{\mathrm{a}}$ \\ a School of Psychology, Bangor University, Wales, UK \\ ${ }^{\mathrm{b}}$ Department of Psychology, University of Hull, Hull, UK
}

\section{A R T I C L E I N F O}

\section{Article history:}

Accepted 22 February 2011

Available online 8 April 2011

\section{Keywords:}

Automatic imitation

Body part priming

Spatial priming

Mirror system

Learning

\begin{abstract}
A B S T R A C T
Observation of another person executing an action primes the same action in the observer's motor system. Recent evidence has shown that these priming effects are flexible, where training of new associations, such as making a foot response when viewing a moving hand, can reduce standard action priming effects (Gillmeister, Catmur, Liepelt, Brass, \& Heyes, 2008). Previously, these effects were obtained after explicit learning tasks in which the trained action was cued by the content of a visual stimulus. Here we report similar learning processes in an implicit task in which the participant's action is selfselected, and subsequent visual effects are determined by the nature of that action. Importantly, we show that these learning processes are specific to associations between actions and viewed body parts, in that incompatible spatial training did not influence body part or spatial priming effects. Our results are consistent with models of visuomotor learning that place particular emphasis on the repeated experience of watching oneself perform an action.
\end{abstract}

\section{Introduction}

Understanding the actions of others and how they relate to our own actions is crucial for successful social interactions. Evidence from behavioural and neuroimaging studies suggests that the observation of others' actions activates similar motor representations in the observer. Hence observing other people perform an action can facilitate our execution of the same action. This is thought to be achieved by means of so called 'mirror systems' in which observed actions are mapped onto one's motor representations of those actions (e.g., Di Pellegrino, Fadiga, Fogassi, Gallese, \& Rizzolatti, 1992; Iacoboni, 2005; but see Hickok, 2009).

The priming of action via observation has been shown to be action specific: the observed action primes the execution of the same action but not a different action performed with the same body part (e.g., Heyes, Bird, Johnson, \& Haggard, 2005; Press, Bird, Walsh, \& Heyes, 2008). Furthermore, there is recent evidence to suggest that these effects are not just action - but also effector specific (e.g., Bach \& Tipper, 2007; Gillmeister et al., 2008). For example, Gillmeister et al. (2008) required participants to lift their hand or their foot in response to a letter presented in the centre of

\footnotetext{
* Corresponding author. Address: School of Psychology, Adeilad Brigantia, Bangor University, Bangor, Wales LL57 2AS, UK. Fax: +44 1248382599.

E-mail address: a.wiggett@bangor.ac.uk (A.J. Wiggett).
}

the screen. If an $\mathrm{H}$ was presented, the participant was required to lift their hand, if an $\mathrm{F}$ was presented they were required to lift their foot. At the same time task-irrelevant stimuli were presented. These were images of a hand and a foot, presented side by side. As the $\mathrm{H}$ or $\mathrm{F}$ was presented, either the hand or foot appeared to lift. The authors found that hand responses were faster when participants observed a hand lifting action on screen, and foot responses were faster when participants observed a foot lifting response on screen.

A key issue concerns whether these associations between sensory inputs and motor outputs are innate (e.g., Meltzoff \& Moore, 1997) or learned, and if the latter, what the conditions for learning are. To address this question, a number of models have been proposed. The associative sequence learning (ASL) model by Heyes and colleagues (Heyes, 2001, 2005; Heyes \& Ray, 2000; Heyes et al., 2005) suggests that the cortical connections mediating motor activation by action observation are formed by correlated experience of observing and executing the same actions. This correlation can arise from self-observation, mirror exposure or synchronous actions with other people (for example being imitated by adults). Critically, this model can account for perceptually transparent movements (e.g. hand movements) as well as perceptually opaque ones (e.g. facial expressions). Keysers and Perrett's (2004) Hebbian model places particular emphasis on self-observation (the repeated experience of watching oneself perform an action) for the emergence of mirror systems. Simultaneously executing and observing an action causes associations to 
be made between the motor output and visual input, such that simply viewing an action becomes sufficient to activate motor representations of that action.

Recent studies have tried to induce incompatible motor sensory associations by using "incompatible" training. The logic of these studies is that if development of mirror systems depends on sensorimotor learning, it should be possible to learn new associations through sensorimotor training (Heyes, 2005). New associations between formally incompatible features of actions may change the functioning of mirror systems and even lead to "countermirror" properties. Furthermore, if new associations between observed and produced actions can be learned, then this predicts changes to the standard action priming effects. For example, Gillmeister et al. (2008); (see also Catmur, Walsh, \& Heyes, 2007) measured effector priming before training and replicated the finding that hand actions were faster when viewing a hand move than when viewing a foot move, and vice versa. The participants were then split into a compatible and an incompatible training group. The compatible group were instructed to lift their hand after seeing a hand lift and to lift their foot after seeing a foot lift. In the incompatible training group this was reversed: the instructions were to execute a hand lift after seeing a foot lift, and to execute a foot lift after seeing a hand lift. After the training phase, participants were tested again on the effector priming task. The results showed that the priming effect was significantly smaller in the incompatible training group compared to the compatible training group. These results suggest that associations between visual and motor features of actions (or "mirror properties") are unlikely to be entirely innate. Rather, the development of the mirror system is driven by correlated sensorimotor learning.

There are various ways in which action production and action perception can become associated. Two central properties of the learning process are: (i) whether learning is implicit or explicit and (ii) the order in which motor output and perceptual input are experienced. First, as an example of the implicit/explicit dimension, learning can be implicit in the sense that an individual has no specific goal to link perception and production of action; or learning could be explicit, where specific motor responses must be produced when viewing specific visual inputs. Second, motor output could be prior to visual perceptual input, or visual input could be prior to the motor output.

In terms of these two features of learning, the studies described above (e.g., Gillmeister et al., 2008) involved explicit learning, and visual perception occurred prior to action production. That is, participants were asked to observe the movement of a visual stimulus (body part) and then make a specific motor response based on what they saw. This form of visuomotor learning is of course exploited in situations of training between people, where an action, for example a golf swing, is executed by an expert and viewed by a novice who then attempts to produce the same action.

However, we propose that the initial associations between motor outputs and visual inputs that form the foundations of mirror systems are likely to be learned quite differently. That is, there is typically no specific goal to be achieved to link perception with action, so learning of this type is normally implicit. Also, in terms of the order, motor efferent output is followed by (and causes) visual afferent input. For example, the newborn infant makes a hand movement with no specific goal and this is followed immediately by visual input of the viewed hand. Both these explicit and implicit forms of learning are clearly part of human learning, but the implicit motor-to-vision form is likely to occur prior to the explicit vision-to-motor form. This is noted in Keysers and Perrett's (2004) Hebbian learning model: the first step in this model is the association between motor commands and visual descriptions of our own actions; the association of observing someone else performing the same action arises subsequently. According to this model, the implicit association of motor and visual aspects of self-generated actions is primary in sensorimotor learning.

The current work attempts to extend the findings of Gillmeister et al. to the more fundamental situation of implicit action production-perception learning. That is, rather than require participants to make specific goal-based motor responses based on viewing particular body part motion (explicit vision-to-action), participants will be required to produce self-initiated actions which will be followed immediately by the perception of specific body movements (Implicit action-to-vision). Similar paradigms have been used to study learned associations between, for example, key presses and tones (e.g., Dutzi \& Hommel, 2009; Elsner et al., 2002; Kunde, 2001; Melcher, Weidema, Eenshuistra, Hommel, \& Gruber, 2008) and between key presses and visually presented stimuli such as words (Hommel, Alonso, \& Fuentes, 2003) or arrows (Kunde, 2004). The learning model proposed by Gillmeister et al., if it is to account for mirror systems, would predict that learning should also take place under these implicit circumstances. In contrast, if no such implicit learning is observed, their learning account would be restricted to situations of explicit learning via action observation and imitation, typically employed in the context of learning specific skills (e.g. dancing, golf swings etc.).

Gillmeister et al. invoke the mirror neuron system to explain their findings. However, other mechanisms may also be contributing - there are clearly many different types of visuomotor stimulus-response ( $\mathrm{S}-\mathrm{R}$ ) effects. As discussed above, seeing an action such as a hand grasping an object facilitates similar actions in an observer. Furthermore, passively viewing an object that is usually acted upon (e.g., hammer) automatically activates motor responses to act on the object (e.g., Tucker \& Ellis, 1998), and the response to a stimulus is faster if the stimulus is close to the hand on the same side of space than when it is on the opposite side (e.g., Simon, 1969). These stimulus-response compatibility effects can have a range of different properties, such as different time-courses (e.g., Hommel, 1994; Phillips \& Ward, 2002). Therefore, as there are a range of different visuomotor priming effects, an open question is whether there is something special about learning associations between making body movements and seeing body movements, or whether these learning processes generalize to other S-R situations.

To test this, we also examined Simon-like spatial stimulus-response effects. Hand and foot responses have been shown to be influenced by spatial compatibilities between stimulus and response (Umiltà \& Nicoletti, 1990; Vu \& Proctor, 2001). That is, hands and feet have associated spatial properties such that the hand is represented as spatially above the foot (as it is normally in the upright posture). Therefore it is predicted that hand responses will be faster when visual primes are presented above the centre of the screen, while foot responses will be faster when the primes are below the centre of the screen, a form of effectorbased vertical Simon effect.

It is not known whether the effect of incongruent effector training generalises to such spatial stimulus-response associations. We address this by examining spatial learning, where for example, a hand response could be associated with a lower, rather than an upper, visual field event. This will allow us to test whether the associations learned between executing body actions and observing body actions constitute special kinds of associations, or whether the effects of incompatible training on S-R compatibilities are more general, extending to spatial codes. Finding effects of incompatible spatial training or a generalisation from one type of training to both types of priming would point towards a generic nature of these learning effects. 


\section{BODY STIMULI}
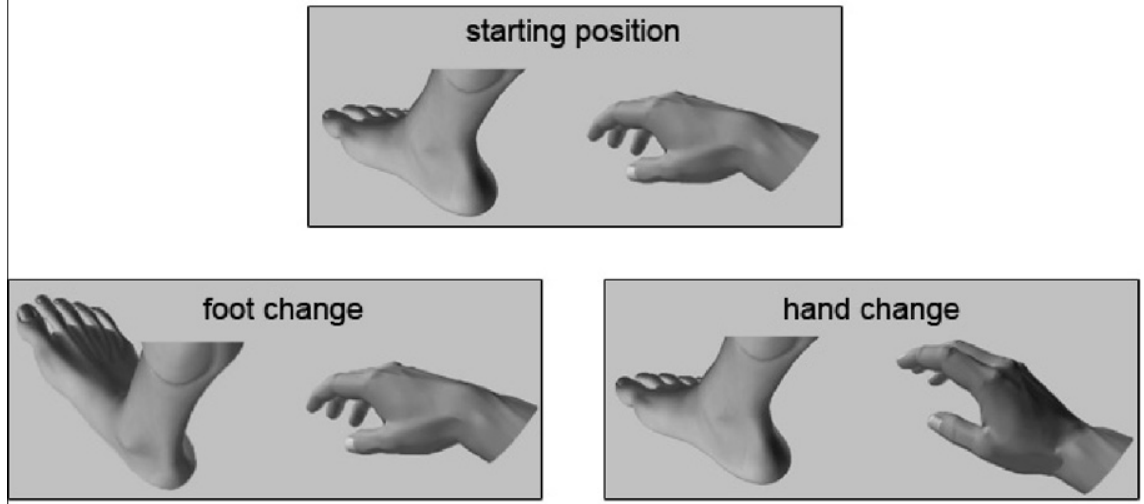

SHAPE STIMULI
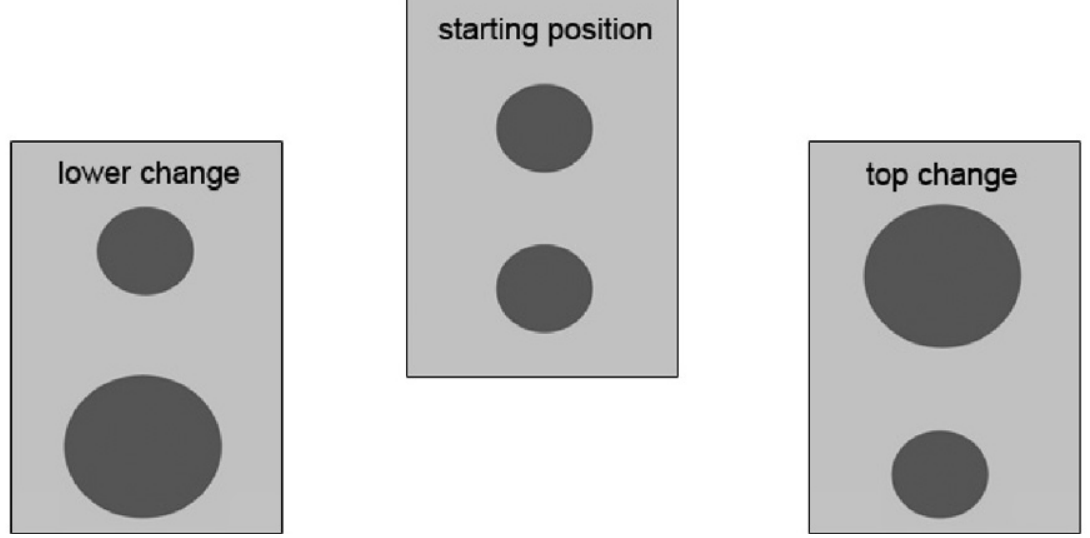

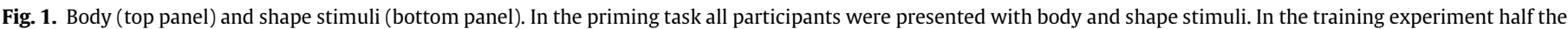
participants saw only body stimuli and half only shape stimuli.

\section{Materials and methods}

\subsection{Participants}

Ninety-six volunteers ( 35 male, 61 female, age range 18-50, mean 22.5 years, $S D=5.1$ years, 87 right handed ${ }^{1}$ ) were either students at Bangor University or recruited from the Bangor community and participated in exchange for course credits or a payment of $£ 6$. Procedures were approved by the Ethics Committee of the School of Psychology at Bangor University.

\subsection{Materials}

Stimuli were presented on a 17-in. LG monitor using MatLab (The MathWorks, MA) and Psychophysics Toolbox (Brainard, 1997; Pelli, 1997), running on an Apple Mac Pro computer. Two types of stimuli were presented in both the priming and the training experiment: body parts (hand, foot) and shapes (circles). Body part stimuli were created using Poser software (Poser, Curious Labs, Santa

\footnotetext{
1 The body compatible group included four left-handed and one ambidextrous participant; the shape compatible group two left-handed participants; the shape incompatible group one left-handed and one ambidextrous participant. Re-analyses of our data leaving out non-right handers did not significantly change the patterns of results.
}

Cruz, CA). In the priming experiment letters were also presented $(\mathrm{H}$ and $\mathrm{F})$ to instruct the participant which movement to execute.

The hand and foot images were from near-egocentric viewpoints (see Fig. 1), presented in greyscale on a grey background. The hand in resting position occupied approximately $11^{\circ}$ of horizontal visual angle and approximately $5^{\circ}$ of vertical visual angle. The foot in resting position occupied approximately $9.5^{\circ}$ of horizontal visual angle and $7^{\circ}$ of vertical visual angle. The lifted hand occupied approximately $11.5^{\circ}$ of horizontal visual angle and approximately $7.5^{\circ}$ of vertical visual angle. The lifted foot occupied approximately $11^{\circ}$ of horizontal visual angle and approximately $9^{\circ}$ of vertical visual angle. Replacing the resting hand/foot with the lifted hand/foot during a trial resulted in an apparent "lifting" motion. The hand and foot stimuli were presented side by side, and the left-right position of the stimuli always stayed the same within a trial. Across trials, the hand and foot were presented as the left or right stimulus with equal frequency. The small circle occupied approximately $4^{\circ}$ of visual angle, the large circle $7^{\circ}$. The circles were presented above and below the centre of the screen. Instruction stimuli consisted of a capital letter ( $\mathrm{H}$ or $\mathrm{F}$ ) presented in black, occupying $1^{\circ}$ of horizontal and $0.8^{\circ}(\mathrm{H})$ and $0.6^{\circ}(\mathrm{F})$ of vertical visual angle.

Response times of hand lifts were measured by recording the release of the space bar; foot responses by recording the release of a foot pedal (Savant Elite FS10 J-USB, Kinesis). Participants used 
their right hand and right foot; the keyboard and foot pedal were positioned slightly to the right of the centre of the screen, allowing a natural, seated body position.

\subsection{Procedure}

All participants completed two kinds of tasks: the priming experiment and the training phase. The experimental session started with two blocks of the priming experiment, followed by four blocks of the training experiment, after which participants completed a further two blocks of the priming experiment. In total the experiment lasted $1 \mathrm{~h}$. Participants sat at a viewing distance of approximately $55 \mathrm{~cm}$ from the monitor. The participant's right hand was placed on the space bar of the keyboard, their right foot on a foot pedal under the desk. The keyboard and the arms of the participant were covered with a custom-made box and attached sheet ensuring that participants could not see either of their hands during the experiment (the foot and foot pedal were also not visible under the desk).

\subsubsection{Priming experiment}

The trial sequence, based closely on Gillmeister et al. (2008), is shown in Fig. 2. Each trial started with a message "Press both keys" indicating to the participant to press the space bar and the foot pedal. Instructions were to keep both pressed until the onset of the letter. Once both keys were pressed, two stimuli were presented - these were either a hand and a foot, or two circles. These stimuli were task-irrelevant. They were shown for a variable duration $(500 \mathrm{~ms}, 750 \mathrm{~ms}, 1000 \mathrm{~ms}, 1250 \mathrm{~ms}$, or $1500 \mathrm{~ms}$, sampled equally), after which either the hand or foot lifted, or one of the two circles increased in size. $175 \mathrm{~ms}$ after this change, a letter was presented in the centre of the screen. This cue instructed the participant to lift their hand $(\mathrm{H})$ or foot $(\mathrm{F})$ as quickly as possible, ignoring the other stimuli on the screen. The letter and body parts/shapes stimuli remained on screen for $2000 \mathrm{~ms}$, after which the message "Press both keys" was presented again.
Two blocks of 96 trials were presented before training, and another two blocks of 96 trials after training. We tested for two types of compatibility effect: body part and spatial (vertical) compatibility. For the body condition, the letters and task-irrelevant stimuli were compatible (e.g. the letter F presented with a foot lift), or incompatible (e.g. a letter $\mathrm{F}$ presented with a hand lift). For the shape condition the letter and the task-irrelevant change could also be spatially compatible or incompatible: the hand was positioned, as normally, above the foot, so that hand movements would be compatible with a change of the top circle, or incompatible with a change of the lower circle. The four different trial types defined by condition (body, shape) and compatibility (compatible, incompatible) were presented equally often and were randomly intermixed within each block. Furthermore, within each condition there were equal numbers of each body/shape change and correct response (hand lift with $\mathrm{H}$, hand lift with $\mathrm{F}$, foot lift with $\mathrm{H}$, foot lift with $F$, top change with $H$, top change with $F$, lower change with $H$, lower change with F). Fig. 3 gives an example of all possible trial types (body compatible, body incompatible, shape compatible, shape incompatible). All participants were tested on all trial types. A short practice block of eight trials was completed before the start of the experimental trials.

\subsubsection{Training phase}

The participants were randomly allocated to one of four training groups: body compatible, body incompatible, shape compatible, or shape incompatible. There were 24 participants in each group. As in the priming experiment, each trial started with the presentation of the "Press both keys" instruction. Once both the space bar and the foot pedal were pressed, a hand and foot (body training groups) or two circles (shape training groups) were presented. These were the same stimuli used in the priming experiment. The stimuli stayed on screen until the participant executed a movement. Participants were instructed to lift their hand or their foot and observe what happened on the screen to detect occasional catch trials (to be described below). On a given trial, the participants could choose which effector to lift; the instructions were to do approximately

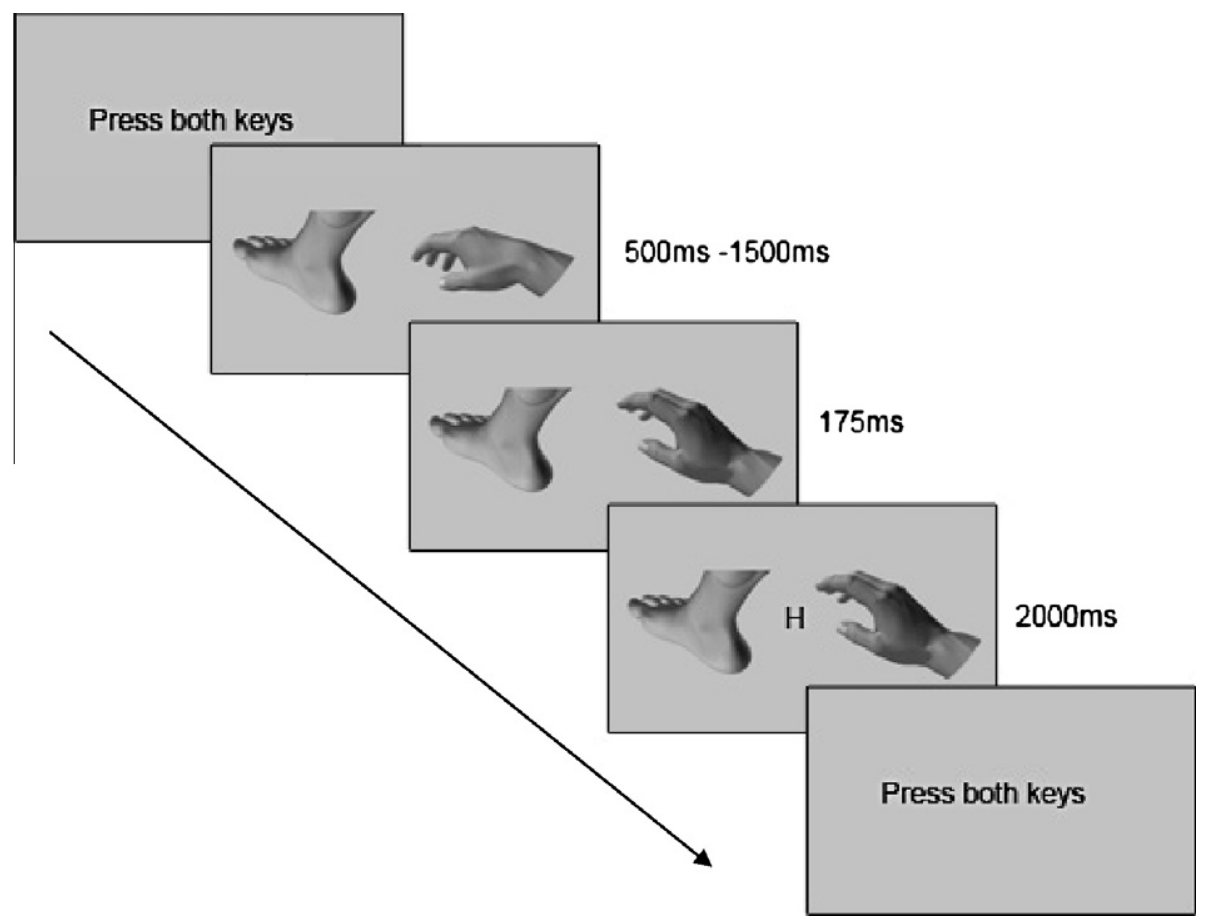

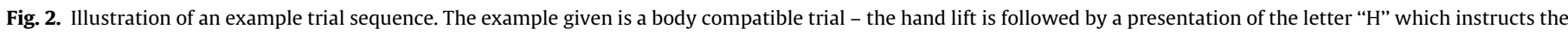
participant to lift their hand. 


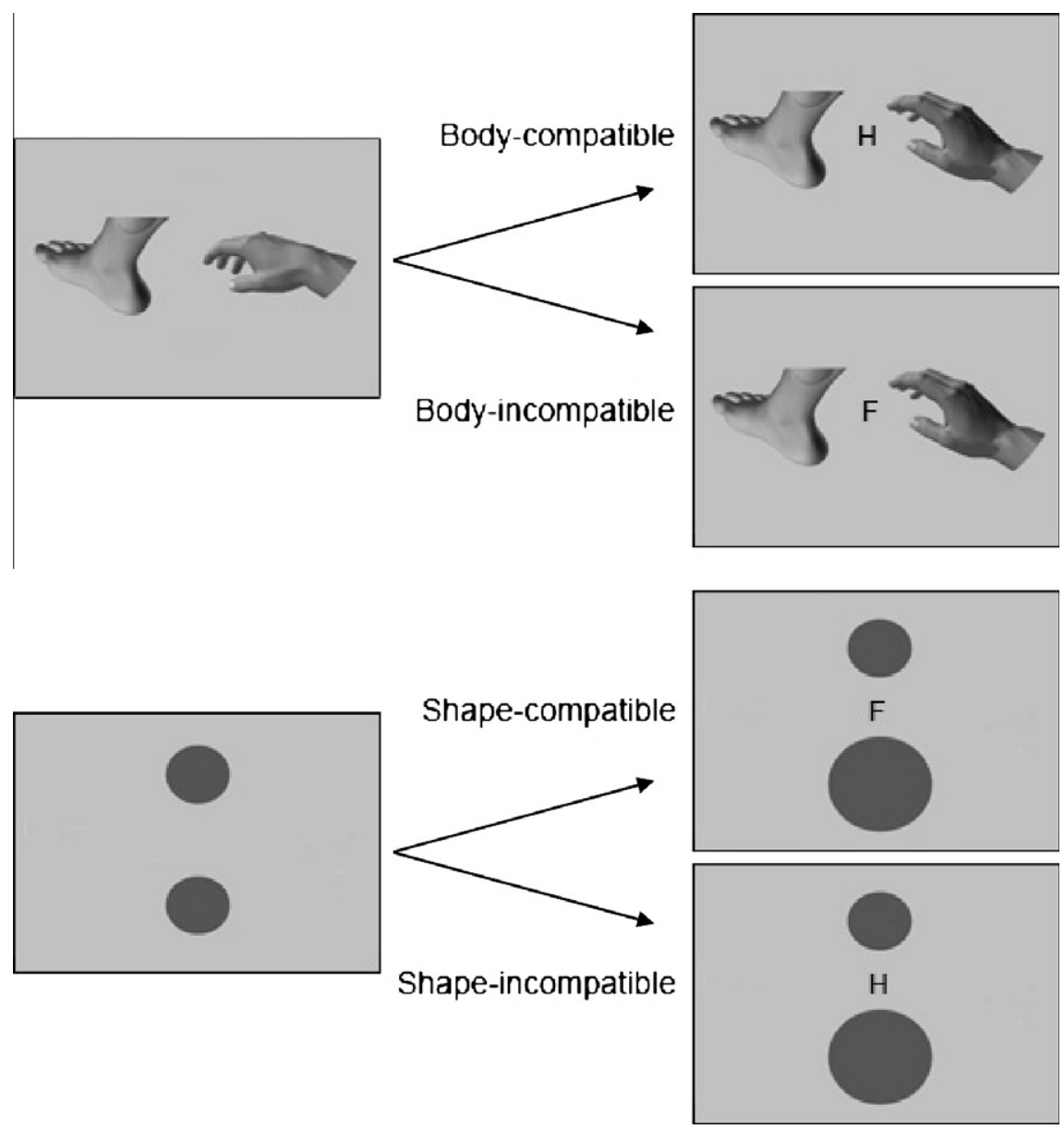

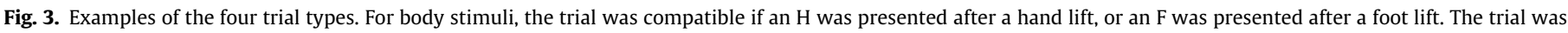

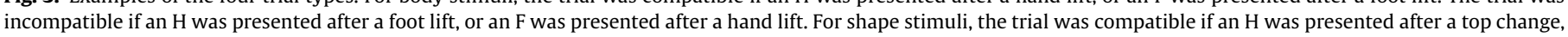
or an $\mathrm{F}$ was presented after a lower change. The trial was incompatible if an $\mathrm{H}$ was presented after a lower change, or an $\mathrm{F}$ was presented after a top change.

equal numbers of hand and foot lifts within each run. Analysis of the frequency of foot and hand lifts indicate that this was achieved (Foot lifts: mean $=46.7 \%$, $S D=3.3 \%$, Hand lifts: mean $53.2 \%$, $\mathrm{SD}=3.3 \%)$. There was no difference across training groups in the number of foot and hand lifts executed (foot: $F(3,92)=0.8$, $p>0.05, \eta_{p}^{2}=0.03$; hand: $\left.F(3,92)=0.77, p>0.05, \eta_{p}^{2}=0.03\right)$.

For the body compatible group, a hand lift resulted in the hand on screen lifting (while the foot stayed the same) and a foot lift resulted in the foot on screen lifting. For the body incompatible group the visual feedback was incongruent with the participant's action: when they lifted their hand, the foot on screen lifted; when they lifted their foot, the hand lifted. For the shape compatible group, a hand lift resulted in a change at the top of the screen (the top circle increased in size), a foot lift resulted in a change at the bottom of the screen (the lower circle increased in size). For the shape incompatible group a hand lift resulted in a change at the bottom of the screen (the lower circle increased in size); a foot lift resulted in a change at the top of the screen (the top circle increased in size). The changes on screen were the only visual feedback the participants had of the visual "consequences" of their movements - they could not see their own hand and feet during the experiment. The change stimuli were on screen for $500 \mathrm{~ms}$, followed by a blank screen for $500 \mathrm{~ms}$, then the next trial started. See Fig. 4 for a summary of the different training conditions. If participants lifted both their hand and their foot simultaneously, no change stimuli were presented and a blank screen was presented for $500 \mathrm{~ms}$.

In order to ensure participants paid attention to the changes on screen a target detection task was introduced: on $5 \%$ of trials the stimulus change was different to the one usually observed. For the body groups, the hand or foot was rotated downwards (the hand/foot in resting position was rotated downwards by $45^{\circ}$ ) rather than upwards. For the shape groups, the circle decreased in size rather than expanding. Participants were instructed to detect these target trials and report them verbally to the experimenter. There were 100 trials per block and participants completed four blocks in total. Participants completed a short practice block of ten trials before starting the training phase. Participants also completed a distractor task (music rating) for approximately 2 min between each block of the training task.

\section{Results}

For six participants in each of the four training groups the error data were not reliably recorded due to a fault in the experiment code. ${ }^{2}$ A table of the average median response times (RTs) and

\footnotetext{
2 If the cue was an ' $\mathrm{H}$ ' and the participant incorrectly lifted the foot, but corrected this error by lifting the hand, the recording of the initial incorrect response was overwritten by the subsequent correction. However, the initial error was not overwritten if participants lifted the hand in response to an ' $\mathrm{F}$ ' and then corrected by lifting the foot. Error rates for these participants were therefore underestimated and they were not included in the analysis of the proportion of errors. However, this affected all training groups equally. Furthermore, the number of errors in response to ' $F$ ' cues for these participants were low (mean $=4.2 \%, \mathrm{SD}=2.0 \%$ ) and so too were the errors in response to ' $\mathrm{H}$ ' cues for the remaining participants ( mean $=5.0 \%, \mathrm{SD}=2.2 \%$ ). As such, the RT data from all participants were included in the analysis of the reaction
} time data. 


\section{Compatible training}

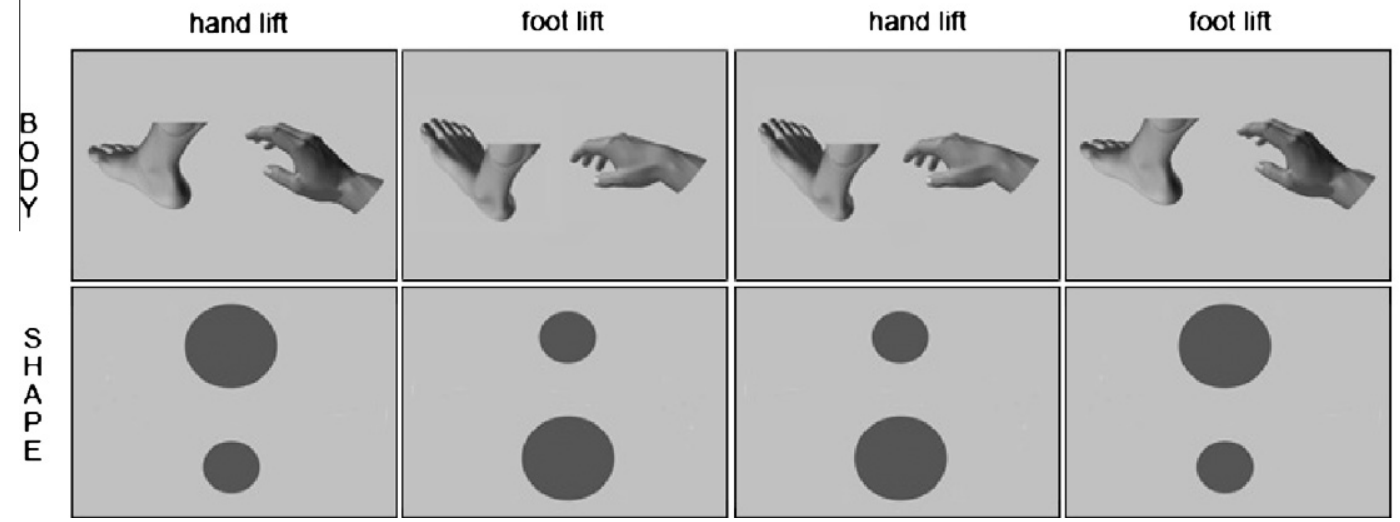

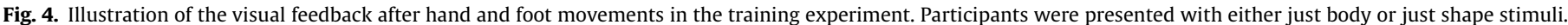

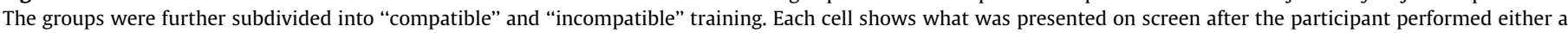
hand or a foot lift.

error rates for each group, for all conditions in the pre- and posttraining sessions is presented in Supplementary Table 1.

\subsection{Pre-training priming effects elicited by effector and spatial compatibility}

We first tested for evidence of effector and spatial compatibility priming prior to training by analysing only the first two blocks of the priming experiment. The median RTs (correct trials only) were entered into a three-way ANOVA with prime stimulus (body part vs. shape) and prime-cue compatibility (compatible vs. incompatible) as within subjects factors, and subsequent training group (body compatible vs. body incompatible vs. shape compatible vs. shape incompatible) as a between subjects factor. There was a significant effect of compatibility, with RTs greater in the incompatible than compatible trials $\left(F(1,92)=177.2, p<0.001, \eta_{p}^{2}=0.66\right)$. There was a significant interaction between prime-cue compatibility and priming stimulus $\left(F(1,92)=34, p<0.001, \eta_{p}^{2}=0.27\right)$, reflecting larger priming effects for body parts than spatial compatibility. However, the effect of compatibility was highly significant for both body $\left(F(1,95)=152.8, p<0.001, \eta_{p}^{2}=0.62\right)$ and shape priming stimuli $\left(F(1,95)=49.8, p<0.001, \eta_{p}^{2}=0.34\right)$. As expected, there was no main effect of training group $(F(3,92)=0.2$, $\left.p>0.05, \eta_{p}^{2}=0.01\right)$, and no interaction of group with any other factor (stimulus $\times$ group: $F(3,92)=0.21, p>0.05, \eta_{p}^{2}=0.01$; compatibility $\times$ group: $\left.F(3,92)=0.73, p>0.05, \eta_{p}^{2}=0.02\right)$.

Therefore, the paradigm employed was successful in eliciting effector priming, thus replicating the findings of previous studies. A priming effect was also elicited by the spatial compatibility of the shape stimuli, albeit not as strongly as for the body stimuli. Furthermore, these priming effects did not differ between the training groups, demonstrating that any modulation after training cannot be attributed to group differences present prior to training.

\subsection{Modulation of priming effects by incompatible training}

Analyses were conducted on the magnitude of the priming effects (incompatible trials minus compatible trials) for the median RTs (correct trials only) and for the proportion of errors. Modulation of the priming effects was predicted to occur in the post-training session as a function of the type of training each group received. That is, the groups who had received training on the body stimuli were expected to differ in the priming effect elicited by body stimuli (but not shape stimuli) in the post-training session.
Likewise, the groups who had received training on the shape stimuli were expected to differ in the priming effect elicited by shape stimuli (but not body stimuli) in the post-training session.

In light of this, a mixed measures ANOVA with weighted contrasts (see Table 1) was conducted. As no difference was expected in several of the conditions (weighted as 1 ), these were collapsed and contrasted with the condition in which an effect of training was hypothesised to occur (weighted as 3 ). This type of analysis is appropriate here as we are specifying a priori the pattern of relationship amongst cell means. The between subjects factor was training group and the within subjects factors were priming session (pretraining vs. post-training), and priming stimulus (body vs. shapes).

\subsubsection{Reaction time analysis}

Mean priming effects (incompatible trials minus compatible trials) for body and shape stimuli in the pre-training and post-training session are shown in Fig. 5. The results are further illustrated and summarised in Fig. 6, which shows the difference between the pre- and post-training priming effects for each group separately for the trained (Panel A) and the untrained stimuli (Panel $B)$. We refer to this figure mainly in the discussion.

The weighted contrast ANOVA revealed a significant interaction between training group and the priming conditions $(F(3,92)=5.38$, $p<0.01, \eta_{p}^{2}=0.15$ ). That is, the priming effect obtained in the predicted condition was significantly different from the priming effect obtained in the remaining conditions, but this depended on the training (body or shape) group. To find the source of this interaction, the weighted ANOVA was conducted separately for the groups who had received training on the body stimuli and the groups who

Table 1

Coefficient matrix for the weighted ANOVA. The contrast weights reflect the predicted effect of training occurring in the post-training priming session in response to body stimuli for the body compatible and incompatible groups, and in response to shape stimuli for the shape compatible and incompatible groups.

\begin{tabular}{llllll}
\hline \multirow{2}{*}{ Training group } & \multicolumn{2}{l}{ Priming conditions } & & \\
\cline { 2 - 3 } & \multicolumn{2}{l}{ Pre-training } & & & \multicolumn{2}{l}{ Post-training } \\
\cline { 2 - 3 } \cline { 5 - 6 } & Body & Shape & & Body & Shape \\
& stimuli & stimuli & & stimuli & stimuli \\
\hline Body compatible & -1 & -1 & & 3 & -1 \\
Body incompatible & 1 & 1 & & -3 & 1 \\
Shape compatible & -1 & -1 & & -1 & 3 \\
Shape incompatible & 1 & 1 & & 1 & -3 \\
\hline
\end{tabular}




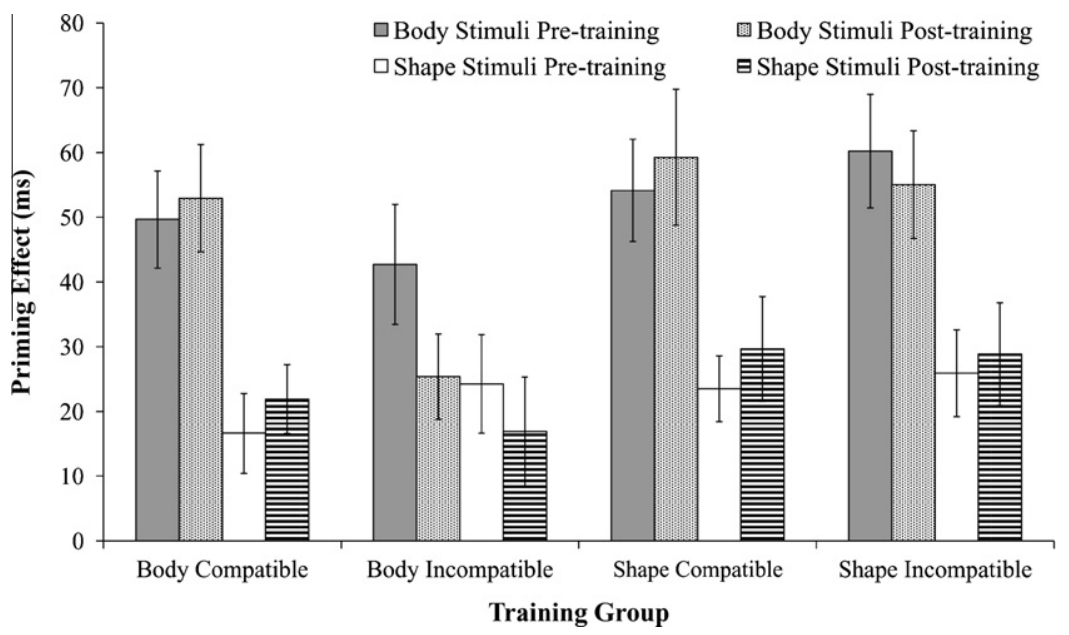

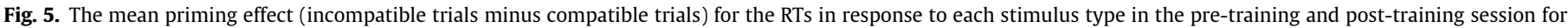
each training group. Error bars represent \pm 1 standard error.

A

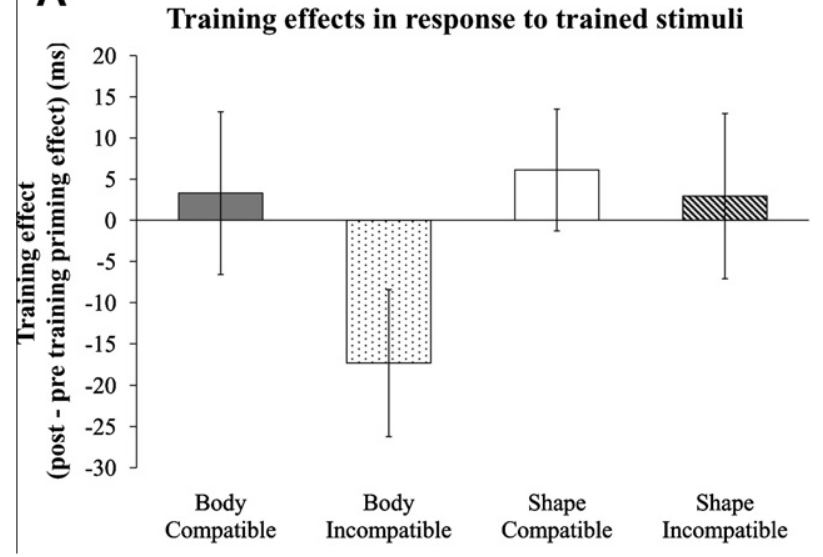

B

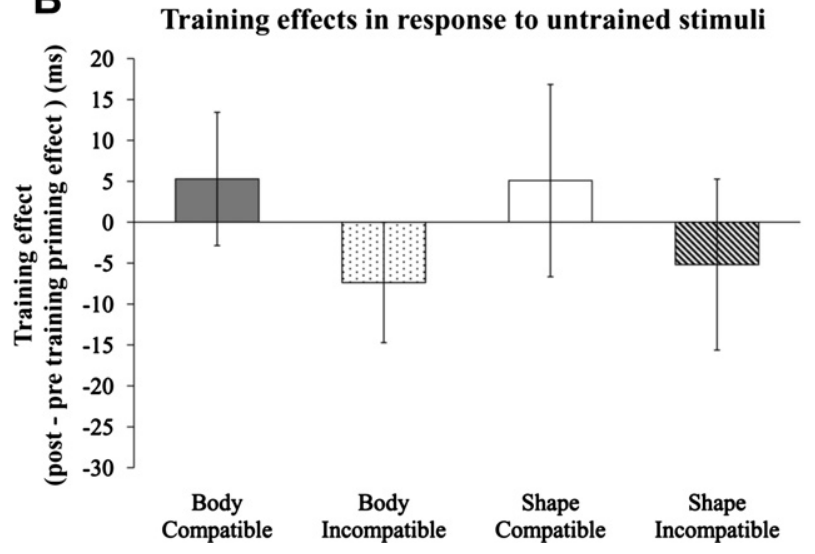

Fig. 6. The modulation of the priming effects as a result of training (post-training priming effect minus the pre-training priming effect). Panel A illustrates the effect of training on the priming effect for each group in response to stimuli for which they had received training. Panel B depicts the effect of training on the priming effect for each group in response to stimuli for which they had not received training. Error bars represent \pm 1 standard error.

had received training on the shape stimuli (the top and bottom halves of the coefficient matrix respectively).

For the body training groups, there was a significant interaction between the priming conditions and the compatibility of their training $\left(F(1,46)=6.08, p<0.05, \eta_{p}^{2}=0.12\right)$. Between subjects AN-
OVAs show that the group who received body incompatible training exhibited a significantly smaller priming effect than the body compatible group in response to body stimuli in the post-training session $\left(F(1,47)=6.76, p<0.05, \eta_{p}^{2}=0.13\right)$. However, there were no group differences in the priming effect for body stimuli in the pre-training session $\left(F(1,47)=0.34, p>0.05, \eta_{p}^{2}=0.01\right)$, nor for the shape stimuli either pre- $\left(F(1,47)=0.6, p>0.05, \eta_{p}^{2}=0.01\right)$ or post-training $\left(F(1,47)=0.25, p>0.05, \eta_{p}^{2}=0.01\right)$.

For the shape training groups, there was no interaction between the compatibility of their training and the priming conditions $\left(F(1,46)=0.03, p>0.05, \eta_{p}^{2}=0.001\right)$. This was confirmed by the absence of group differences in the priming effect elicited by any of the priming conditions (shape stimuli/pre-training: $F(1,47)=$ $0.08, p>0.05, \eta_{p}^{2}=0.002$; shape stimuli/post-training: $F(1,47)=$ $0.01, p>0.05, \quad \eta_{p}^{2}=0.0001$; body stimuli/pre-training: $F(1,47)$ $=0.27, p>0.05, \eta_{p}^{2}=0.01$; body stimuli/post-training: $F(1,47)=$ $0.1, p>0.05, \eta_{p}^{2}=0.002$ ).

We performed a series of exploratory post hoc tests to address the question of whether the effects of incongruent body part training decay over time. To do this we analysed the first and the second post-training priming blocks separately and tested whether there was a significant difference in the body part priming effect between the two training groups (compatible body, incompatible body). The results suggest that the effects are indeed transient: immediately after training (in the first post-training block) the priming effect was significantly smaller for the incompatible compared to the compatible training group $(t(46)=2.5, p<0.05)$. However, by the second block there was no difference between the two groups $(t(46)=0.755, p>0.05)$. To check that this was not due to a general effect of fatigue on compatibility over time, we tested whether the compatibility effect for body stimuli (across groups) significantly changed over the four blocks. The main effect of compatibility was highly significant $(F(1,94)=201.62, p<0.001$, $\left.\eta_{p}^{2}=0.68\right)$, and the main effect of block was marginally significant $\left(F(3,282)=2.61, p=0.052, \eta_{p}^{2}=0.03\right)$. However there was no interaction between block and compatibility $(F(3,282)=0.43$, $p>0.05, \eta_{p}^{2}=0.005$ ). This suggests that, while the effects of incompatible training decayed, the compatibility effects across groups persisted over time.

We repeated the analysis of first and second post-training blocks for the shape training groups, testing whether there was a significant difference in the spatial priming effect between the congruent and the incongruent shape training groups in either block. However, no hint of a difference was found in either the first 
post-training block $(t(46)=0.83, p>0.05)$ or the second post-training block $(t(46)=0.19, p>0.05)$.

\subsubsection{Error analysis}

The proportion of errors reveals a pattern of priming effects that is consistent with the obtained effects in reaction times. However, the weighted contrasts ANOVA revealed no significant interaction between training group and priming condition $(F(3,68)=2.573$, $\left.p>0.05, \eta_{p}^{2}=0.1\right)$. Because the interaction approached significance $(p=0.06)$, further analyses were conducted on the body training groups and the shape training groups separately. However, neither the groups trained on the body stimuli $(F(1,34)=0.71, p>0.05$, $\left.\eta_{p}^{2}=0.02\right)$ nor the groups trained on the shape stimuli $(F(1,34)=$ $0.24, p>0.05, \eta_{p}^{2}=0.01$ ) showed an interaction between the priming conditions and the compatibility of their training.

\subsection{Transfer effects}

It is clear from the above analyses that incompatible spatial training had no effect on either type of priming. However, the magnitude of the spatial priming effect seems to be reduced in the group of participants that underwent incompatible body training (Fig. 6, Panel B "Body Incompatible"). In other words, there appears to be a trend in the data suggesting that the effect of incompatible body training may have generalised or transferred to spatial priming. This is supported by a by non-significant stimulus (body, shape) $\times$ training (pre, post) interaction within the incompatible body training group $\left(F(1,23)=0.66, p>0.05, \eta_{p}^{2}=0.03\right)$. To test whether this was evidence for significant learning transfer, we carried out two additional analyses. Firstly, the equivalent ANOVA for the two body training groups weighted in favour of the post-training shape stimuli condition (instead of the post-training body stimuli as reported above) showed no significant interaction between group and priming condition $\left(F(1,46)=0.14, p>0.05, \eta_{p}^{2}=0.003\right)$. Secondly, we tested whether the size of the change in the two priming effects was correlated within subjects. The logic of this analysis is that if transfer of learning has taken place, then subjects who show a decrease in body part priming should also show a decrease in spatial priming. However, there was no relationship between body part and spatial priming within individual subjects in the incompatible body training group (Pearson's $r=-0.14, p>0.05$ ). This suggests that the change in the size of the spatial priming effect is not related to the change in body part priming in this group of participants.

\section{Discussion}

Previous research (e.g., Gillmeister et al., 2008) has shown that viewing a body part executing an action (such as a hand movement) can facilitate subsequent actions with the same body part. Such findings support notions of the close links between the production and the perception of action, where visual inputs and motor outputs converge. A key issue is whether such vision-action relationships are innate, or whether they are flexible, emerging from learning processes. Gillmeister et al.'s findings provide evidence for the learning hypothesis by showing that after learning incompatible associations, where for example observation of a hand response required the production of a foot response, action priming effects are significantly reduced.

In the current study we have engaged with two further issues. In the training phase of Gillmeister et al.'s study, participants were given explicit instructions to analyse a visual body movement, and either produce an action with the same body part (compatible) or a different body part (incompatible). This can be described as "explicit stimulus-response" learning. Our study examines the alterna- tive situation, where the associations between vision and action are implicit, and action produces subsequent visual consequences (i.e., "implicit response-stimulus" learning). This latter situation is fundamental to visuomotor learning, where the brain learns to build associations between motor commands and subsequent sensory feedback, enabling prediction of the sensory consequences of motor commands (e.g., Shadmehr \& Krakauer, 2008; see also Hommel, 2009). Thus in the training phase our participants freely produced an action, and then observed subsequent visual events. The second main issue we considered within the current design was whether these action-vision learning processes are specific to the observation of body parts, or whether similar effects are found with spatial stimulus-response compatibilities.

We were successful in obtaining priming effects both in response to the effector compatibility of an action and in response to the spatial compatibility of a shape stimulus. Furthermore, the extent of the respective priming effects was selectively modulated as a function of the compatibility of training and the stimulus in which they were trained. The priming effect in response to viewing body parts was smaller for those who had received incompatible training with body stimuli compared to those who had received compatible training. In contrast, the priming effect elicited by spatial compatibility was not modulated by training: there was no difference between the compatible and the incompatible groups who had received training on the shape stimuli.

Fig. 6 provides a summary of the core data, providing answers to our two main research questions. This figure shows the change in priming effect from those obtained in the baseline pre-training conditions with the priming effects obtained after training. The top panel (A) plots the change in the size of the priming effect for the trained stimuli. For example, when the training is done on body parts, what is the effect on body priming? The lower panel (B) shows the effects of training on the priming effect involving the untrained stimuli. For example, after training on response-spatial stimulus associations (shape training), what is the effect on body priming effects?

When considering the left side of Panel A, it is clear that when motor-visual training involving body stimuli is compatible (e.g. a hand movement produces a subsequent visual hand movement), later body priming effects remain constant. However, most importantly, when the body training is incompatible (e.g. a hand movement produces a subsequent movement of a visual foot), the later body priming effect is reduced relative to pre-training priming effects. Furthermore, the right side of Panel A shows that this Training effect is not observed for spatial dimensions after training with the shape stimuli.

In terms of the second issue of learning transfer, consideration of Panel B shows that learning effects do not transfer to other domains. For example, shape incompatible spatial training had no effect on subsequent body compatibility priming effects etc. These results support the notion that the effects of incompatible body training found here, and in Gillmeister et al. (2008), are specific to learning motor-visual associations involving the body rather than more general effects of incompatible stimulus-response training.

In line with Gillmeister et al. our findings support the notion that learning of new motor-visual associations can take place in a range of conditions (Heyes, 2001; Heyes, 2005). In the training phase of the current study the action was self-determined, and the subsequent visual observation of body movement was determined by the action. We believe this closely matches the situation of the early development of visuomotor systems, and the fine-tuning of motor systems throughout life. For example, infants activate motor systems to make a hand movement and immediately see the effect of this motor command, as they view their own hand move. We suggest that these implicit learning processes are likely to be 
the primary factor in the development of mirror cells (Keysers \& Perrett, 2004), and occur prior to later explicit learning, where an individual explicitly copies an action of another person. Other types of sensory-motor experiences, such as being imitated while performing an action, or engaging in synchronous actions in response to a common external stimulus, are also likely to occur before explicit learning by imitation can take place. Of course all these types of learning are likely to be important sources of experience contributing to the further development and strengthening of the mirror system (Heyes, 2001; Heyes \& Ray, 2000).

In some senses, these motor learning effects are quite remarkable. After producing a motor response, the subsequent visual effect of a body movement is incidental, in that participants are not explicitly associating motor output with sensory feedback, but are merely monitoring the visual inputs for occasional oddball targets. Nevertheless, in a relatively short period of time, the effects of an entire lifetime of motor-visual associations can be reduced to some extent. However, one might assume that these learning effects would be somewhat transient, decaying if not actively maintained. Analysis of the first and second post-training priming blocks confirmed this. That is, immediately after training there was a significant difference, where the priming effects in the incompatible training group were less than those in the compatible training group. In constrast, by the second block, prior compatible/incompatible training had no effects. The transient nature of our effects contrasts with that of Gillmeister et al. (2008), where effects were observed $24 \mathrm{~h}$ after training. However, at this time we do not know whether this contrast is due to the implicit Vs explicit nature of the learning, or due to the more extensive learning period (days) in the Gillmeister et al. study.

The issue concerning the generality of the learning effect is reflected in the right side of Panel A in Fig. 6. It is clear that compatible or incompatible training of the motor-visuospatial associations has no effect on the subsequent spatial priming effects. Thus after a series of incompatible training trials, where a hand response consistently causes the lower stimulus to expand, for example, the subsequent priming effects remain constant: a hand response is facilitated/primed by a change in the upper stimulus just prior to the response, and a foot response is facilitated/primed by a change in the lower stimulus. Our results suggest that this is the case even immediately after incompatible shape training (i.e. in the first posttraining block of the priming experiment).

As noted above, there are many kinds of vision-action priming effects. Some of these are related to object identity, such as the body part priming described here (Gillmeister et al., 2008), and object affordances (Tucker \& Ellis, 1998); while others are not related to objects, but are spatial in nature (Simon, 1969; Vu \& Proctor, 2001; Vu, Proctor, \& Pick, 2000). These different effects do not always have the same properties: for example, spatial Simon effects are fast and transient, whereas object affordance effects can be slower to emerge and be more stable (Phillips \& Ward, 2002). With the findings of the current study we have identified another way in which these kinds of effects differ: our results suggest that body related learning is flexible, whereas the more basic spatial relationships between action and perception are less flexible, and not so easily re-trained.

It has been proposed that shifts in spatial attention mediate spatial compatibility effects (e.g., Nicoletti \& Umiltà, 1994), where the onset of a stimulus, relative to the focus of attention, determines S-R compatibility effects. Thus, the sudden onset stimulus in the periphery triggers rapid exogenous orienting to its location, and this spatial shift of attention produces action priming. Because such exogenous shifts of attention are fast and automatic, mediated by mid-brain structures such as the superior colliculus, they are less likely to be altered via learning. That is, a sudden onset expansion of a stimulus in the upper half of the screen will trigger a rapid attention shift, and this basic vision-action link cannot be re-mapped, where the upper stimulus triggered a rapid attention shift to the opposite lower side of the screen. Of course we cannot exclude that other action-vision training regimes might produce learning effects for spatial dimensions. Indeed, it has been shown that in some situations the Simon effect can be reduced after explicit, incompatible spatial training (Tagliabue, Zorzi, Umiltà, \& Bassignani, 2000; Vu, 2007).

In summary, the current study has revealed two things. Firstly, our results show that there is flexibility in some vision-action systems. Observing a body in action primes similar actions in the viewer, but this is flexible in the sense that different action-perception associations involving the body can be established via learning. Most importantly, such learning effects can emerge implicitly, i.e. when internally generated actions produce visual effects, where no specific goal is specified, and after a relatively brief training period. We propose that this form of learning would seem to be key to the emergence of mirror cells, and hence is important for theories postulating that mirror cell systems emerge from learning, rather than being innately pre-specified. However, we appreciate that this is a controversial speculation and hence further research will be necessary. The second finding is that spatial action priming effects are not modulated by incompatible spatial learning, and the type of action-vision learning that is related to the body does not appear to generalize to spatial priming effects. Our results support the view that associations between executing and observing body actions constitute special kinds of learned associations.

\section{Acknowledgments}

This research was supported by the Economic and Social Research Council (Res-062-23-0980).

\section{Appendix A. Supplementary material}

Supplementary data associated with this article can be found, in the online version, at doi:10.1016/j.bandc.2011.02.014.

\section{References}

Bach, P., \& Tipper, S. P. (2007). Implicit action encoding influences personal-trait judgements. Cognition, 102, 151-178.

Brainard, D. H. (1997). The psychophysics toolbox. Spatial Vision, 10, 433-436.

Catmur, C., Walsh, V., \& Heyes, C. (2007). Sensorimotor learning configures the human mirror system. Current Biology, 17, 1527-1531.

Di Pellegrino, G., Fadiga, L., Fogassi, L., Gallese, V., \& Rizzolatti, G. (1992). Understanding motor events: A neurophysiological study. Experimental Brain Research, 91, 176-189.

Dutzi, I. B., \& Hommel, B. (2009). The microgenesis of action-effect binding. Psychological Research, 73, 425-435.

Elsner, B., Hommel, B., Mentschel, C., Drzezga, A., Prinz, W., Conrad, B., et al. (2002). Linking actions and their perceivable consequences in the human brain. Neuroimage, 17, 364-372.

Gillmeister, H., Catmur, C., Liepelt, R., Brass, M., \& Heyes, C. (2008). Experiencebased priming of body-parts: A study on action imitation. Brain Research, 1217, 157-170.

Heyes, C. M. (2001). Causes and consequences of imitation. Trends in Cognitive Sciences, 5, 253-261.

Heyes, C. (2005). Imitation by association. In S. Hurley \& N. Chater (Eds.) Perspectives on imitation: From mirror neurons to memes (pp. 157-176). Cambridge, Massachusetts: MIT Press.

Heyes, C., Bird, G., Johnson, H., \& Haggard, P. (2005). Experience modulates automatic imitation. Cognitive Brain Research, 22, 233-240.

Heyes, C., \& Ray, E. (2000). What is the significance of imitation in animals? Advances in the Study of Behavior, 29, 215-245.

Hickok, G. (2009). Eight problems for the mirror neuron theory of action understanding in monkeys and humans. Journal of Cognitive Neuroscience, 21, 1229-1243.

Hommel, B. (1994). Spontaneous decay of response code activation. Psychological Research, 56, 261-268.

Hommel, B. (2009). Action control according to theory of event coding (TEC). Psychological Research, 73, 512-526. 
96

A.J. Wiggett et al./Brain and Cognition 76 (2011) 87-96

Hormel, B., Alonso, D., \& Fuentes, L. J. (2003). Acquisition and generalization of action effects. Visual Cognition, 10, 965-986.

Iacoboni, M. (2005). Neural mechanisms of imitation. Current Opinion in Neurobiology, 15, 632-637.

Keysers, C., \& Perrett, D. I. (2004). Demystifying social cognition: A Hebbian perspective. Trends in Cognitive Sciences, 8, 501-550.

Kunde, W. (2001). Response-effect compatibility in manual choice reaction tasks. Journal of Experimental Psychology: Human Perception and Performance, 27, 387-394.

Kunde, W. (2004). Response priming by supraliminal and subliminal action effects Psychological Research, 68, 91-96.

Melcher, T., Weidema, M., Eenshuistra, R. M., Hormel, B., \& Gruber, O. (2008). The neural substrate of the ideomotor principle: An event-related fMRI analysis. Neuroimage, 39, 1274-1288.

Meltzoff, A. N., \& Moore, M. K. (1997). Explaining facial imitation: A theoretical model. Early Development \& Parenting, 6, 79-192.

Nicoletti, R., \& Umiltà, C. (1994). Attention shifts produce spatial stimulus codes. Psycholological Research, 56, 144-150.

Pali, D. G. (1997). The VideoToolbox software for visual psychophysics: Transforming numbers into movies. Spatial Vision, 10, 437-442.

Phillips, J. C., \& Ward, R. (2002). S-R correspondence effects of irrelevant affordances: Time course and specificity of response activation. Visual Cognition, 9, 540-558.

Press, C., Bird, G., Walsh, E., \& Heyes, C. (2008). Automatic imitation of intransitive actions. Brain and Cognition, 67, 44-50.
Shadmehr, R., \& Krakauer, J. W. (2008). A computational neuroanatomy for motor control. Experimenal Brain Research, 185, 359-381.

Simon, J. R. (1969). Reactions towards the source of a stimulus. Journal of Experimental Psychology, 81, 174-176.

Tagliabue, M., Zorzi, M., Umiltà, C., \& Bassignani, F. (2000). The role of long-termmemory and short-term-memory links in the Simon effect. Journal of Experimental Psychology: Human Perception and Performance, 26, 648-670.

Tucker, M., \& Ellis, R. (1998). On the relationship between seen objects and components of potential actions. Journal of Experimental Psychology: Human Perception and Performance, 24, 830-846.

Umiltà, C., \& Nicoletti, R. (1990). Spatial stimulus-response compatibility. In R. W. Proctor \& T. G. Reeve (Eds.). Stimulus-response compatibility: An integrated perspective. Advances in psychology (Vol. 65, pp. 89-116). Oxford, England: North-Holland.

Qu, K.-P. L. (2007). Influences on the Simon effect of prior practice with spatially incompatible mappings: Transfer within and between horizontal and vertical dimensions. Memory E Cognition, 35, 1463-1471.

Wu, K.-P. L., \& Proctor, R. W. (2001). Determinants of right-left and top-bottom prevalence for two-dimensional spatial compatibility. Journal of Experimental Psychology: Human Perception and Performance, 27, 813-828.

Wu, K.-P. L., Proctor, R. W., \& Pick, D. F. (2000). Vertical versus horizontal compatibility: Left-right prevalence with bimanual keypresses. Psychological Research, 64, 25-40. 